\title{
Smaller critical size and enhanced strength by nano-laminated structure in nickel
}

\author{
Wen Wang, Fuping Yuan*, Xiaolei Wu \\ State Key Laboratory of Nonlinear Mechanics, Institute of Mechanics, Chinese Academy of Science, Beijing 100190, People's Republic of China
}

\section{A R T I C L E I N F O}

\section{Article history:}

Received 22 April 2015

Received in revised form 26 June 2015

Accepted 2 August 2015

\section{Keywords:}

Molecular dynamics

Dislocations

Grain boundaries

Laminated structure

Equiaxed grain structure

\begin{abstract}
A B S T R A C T
Because of a shift in the dominant deformation mechanisms, the strength/hardness of metals increases with decreasing grain size down to a critical value, then decreases with further grain refinement. Here, laminated structure with low-angle grain boundaries was found to have smaller critical size and enhanced strength when compared to the equiaxed grain structure, through a series of large-scale molecular dynamics simulations. Then, the corresponding atomistic mechanisms were investigated by checking and comparing the rotations of grains and equivalent strain partitioning in grain boundary atoms. In laminated structure, grain boundary activies were found to be promoted with decreasing lamellar thickness. More importantly, when compared to the equiaxed grain structure at the same length scale (lamellar thickness/grain size), grain boundary activities were found to be inhibited by the laminated structure, which is the main reason for the enhanced strength and the smaller critical size. Besides grain boundary activities, formation of extended dislocations, formation of deformation twins, partial dislocations interacting with formed TBs, partial dislocation blocking by and even transmission through low-angle grain boundaries were also found to play important roles in plastic deformation of nano-laminated structure. The current findings should provide insights for designing stronger and more stable nanostructured metals.
\end{abstract}

(c) 2015 Published by Elsevier B.V.

\section{Introduction}

High strength can be achieved in metals traditionally by introducing more grain boundaries (GBs) via grain refinement or by increasing dislocation density in grain interior [1-8]. Because of a shift in the dominant deformation mechanisms from dislocationmediated plasticity to GB-associated plasticity, the strength/hardness of metals has been found to increase first with decreasing grain size down to a critical grain size $(10-20 \mathrm{~nm})$, and then decrease with further grain refinement [1,2,9-15]. Moreover, a similar trend for twinboundary spacing (TBS) effect on the strength of nanotwinned (NT) metals was also found due to a transition of deformation mechanisms [16-20].

The effects of length scale (layer thickness) on the strength of bi-metal multilayered laminates have also been studied from the micrometer to the nanometer range [21-27]. The strengthening effects as a function of layer thickness can be summarized as three mechanisms: (i) the dislocation pile-up mechanism from submicron to micron range; (ii) the confined layer slip mechanism from few nm to a few tens of nm range; (iii) the interface crossing

\footnotetext{
* Corresponding author.

E-mail address: fpyuan@lnm.imech.ac.cn (F. Yuan).
}

mechanism for about $1-2 \mathrm{~nm}[21,22]$. The strength of bi-metal multilayered laminates increases with decreasing layer thickness in the range of the first two mechanisms and decreases with further reduced layer thickness in the range of the third mechanism. The strongest layer thickness in bi-metal multilayered laminates was found to be less than $5 \mathrm{~nm}[21,22]$, and this critical length scale is much smaller than the critical grain size observed for nanocrystalline metals. Recently, nanostructured metals with laminated grain structure (with aspect ratio ranging from a few to a few tens) and low-angle grain boundaries (LAGBs) could be produced by severe plastic deformation (SPD), such as accumulative roll-bonding (ARB) [3], equal channel angular pressing/extrusion (ECAP/ECAE) [4,28], and surface mechanical grinding treatment (SMGT) [29]. These nanostructured metals with laminated grain structure were observed to be ultrahard and ultrastable $[3,4,28,29]$, while the strongest critical lamellar thickness (the length scale controlling strength) and the underlying deformation mechanisms are still open to further investigations, especially when compared to the equiaxed grain structure.

Due to the inherent limitations, the strain rate is typically high $\left(>10^{7} / \mathrm{s}\right)$ in MD simulations, and some deformation behaviors (such as diffusion effects) might be limited at such short deformation times at ambient temperatures. However, MD simulations with 
carefully designed model system not only have been shown to be particularly useful for studying the plastic deformation mechanism (dislocation activities, GB sliding, GB migration and grain rotation) in nanostructured metals, in which the "in-situ" deformation processes of the system and the stress and the strain distributions at atomic-level can be examined [9,17,30-36], but also have proven to be useful for investigating the size effects (grain size/TBS) on the strength of nanostructured metals $[9,17]$. In this regard, the focus of this paper is to find out and compare the strongest critical length scale (lamellar thickness/grain size) and the underlying atomistic deformation mechanisms for both laminated structure and equiaxed grain structure using a series of large-scale MD simulations.

\section{Simulation procedures}

The Large-scale Atomic/Molecular Massively Parallel Simulator (LAMMPS) code was used for the MD simulations. The force interactions between atoms were described by a widely used Ni EAM potential developed by Mishin et al. [37]. In order to explore the plastic deformation process of the laminated structure with large size (the maximum investigated lamellar thickness was $25 \mathrm{~nm}$ with aspect ratio of 10 in the present study), it is necessary to consider simulation cells larger than those possible in fully 3dimensional systems. Quasi 3-dimensional simulations with a columnar grain structure were considered in this regard, very similar to the configuration proposed by Yamakov et al. [30]. As indicated in the previous research $[17,30]$, the columnar model could also be used to investigate the size effects (grain size or twin boundary spacing) on the strength of nanostructured metals although all dislocation lines are constrained to be parallel to the columnar axis. The lamellar thickness for laminated structure is noted as $\lambda$, while the grain size for equiaxed grains is noted as $d$ here. The thickness direction ( $z$ direction) is along [ 110$]$ and contains 12 atomic planes. The relaxed $\mathrm{Ni}$ samples with laminated structure (lamellar thickness $\lambda=10 \mathrm{~nm}$ ) and equiaxed grains (grain size $d=10 \mathrm{~nm}$ ) are shown in Fig. 1(a) and (b), respectively. The grains of 2,3 , and 4 are rotated from grain 1 about $[\overline{1} 10]$ axis with the angles of $-5^{0}, 5^{0}$, and $10^{\circ}$ respectively for both structures,

(a)

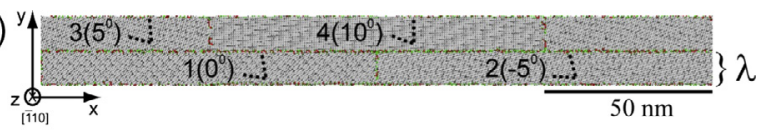

(b)

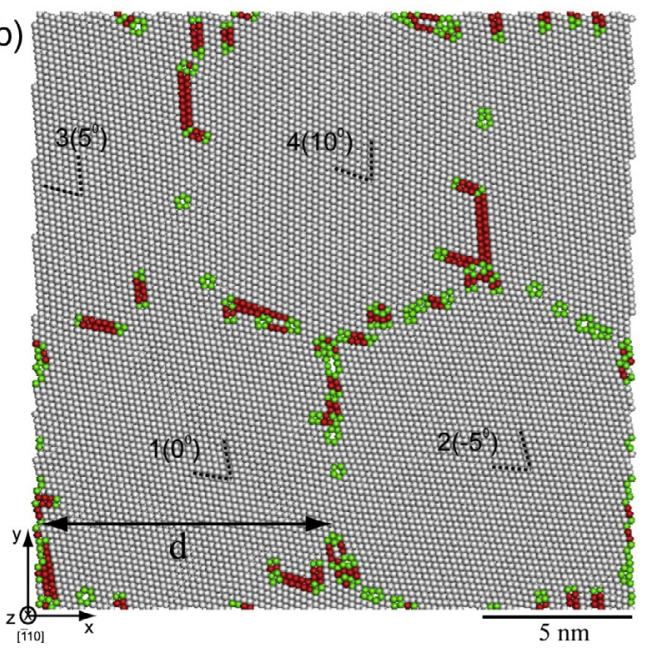

Fig. 1. (a) The relaxed $\mathrm{Ni}$ sample with the laminated structure $(\lambda=10 \mathrm{~nm})$. (b) The relaxed Ni sample with the equal-axed grain structure $(d=10 \mathrm{~nm})$. which generates low-angle boundaries for all grains. The GBs were determined by Voronoi construction in the present study (in the laminated structures, the grain shape was determined only by surrounding four seeds instead of six seeds in the equiaxed grains). Also the dash lines are indications of the orientations of the two sets of $\{111\}$ slip planes in each grain. In the present study, the atoms are painted with colors according to common neighbor analysis (CNA) values. The CNA is a very useful tool used in MD simulations which allows us to determine the local ordering and the defects in a given structure. Perfect fcc atoms are painted by gray color, hcp atoms are painted by red color and green color stands for other atoms which belong to GBs, dislocation cores, free surfaces or other defects.

It should be noted that the LAGBs are actually dislocation walls consisting of discontinuous dislocations due to small misorientation angles. For laminated structure, eight samples with initial lamellar thickness of $\lambda=5 \mathrm{~nm}, 7.5 \mathrm{~nm}, 10 \mathrm{~nm}, 12.5 \mathrm{~nm}, 15 \mathrm{~nm}$, $17.5 \mathrm{~nm}, 20 \mathrm{~nm}$, and $25 \mathrm{~nm}$ were simulated, and the aspect ratio was fixed at 10 (in the range of typical nano-laminates). The dimensions are $500 \times 50 \times 1.49 \mathrm{~nm}^{3}$ for the sample of laminated structure with $\lambda=25 \mathrm{~nm}$, which contains approximately $3,500,000$ atoms. For equiaxed grain structure, eight samples with initial grain size of $d=5 \mathrm{~nm}, 10 \mathrm{~nm}, 15 \mathrm{~nm}, 17.5 \mathrm{~nm}, 20 \mathrm{~nm}$, $22.5 \mathrm{~nm}, 25 \mathrm{~nm}, 30 \mathrm{~nm}$ were considered. The dimensions are $60 \times 60 \times 1.49 \mathrm{~nm}^{3}$ for the sample of equiaxed grain structure with $d=30 \mathrm{~nm}$, which contains approximately 490,000 atoms. When the lamellar thickness/grain size was changed, the same structure and the same crystallographic orientations of all grains were retained. Periodic boundary conditions were used in all three directions, and the tensile loading was along $x$ direction. By the conjugate gradient method, the as-constructed samples were first subjected to energy minimization before the tensile loading, and then heated up to the desired temperature and finally relaxed by the Nose/Hoover isobaric-isothermal ensemble (NPT) under both the pressure 0 bar and the desired temperature $(10 \mathrm{~K})$ for $100 \mathrm{ps}$. A strain of $8 \%$ was applied to each sample (with a constant engineering strain rate of $5 \times 10^{8} \mathrm{~s}^{-1}$ ) after the relaxation. In order to simulate the uniaxial loading, the pressures in the $y$ and $z$ directions were kept to zero during the tensile loading.

\section{Results and discussions}

In samples with equiaxed grains, the strength is determined by grain size. However, the lamellar thickness should be the controlling length scale for strengthening in samples with laminated structure (especially with large aspect ratio, such as 10 used in the present study). Fig. 2(a) and (b) shows the stress-strain curves for samples with laminated structure and equiaxed grains, respectively. Tensile stresses are observed to deviate from linear relationship with strain at a certain stress, which is an indication of onset of plastic deformation, and then gradually reach to a relatively steady-state value regardless of $\lambda / d$.

It is typically more meaningful to compare the average flow stress over a certain plastic strain interval for illustrating the effect of $\lambda / d$ on the flow strength in MD simulations $[9,12,17,19]$. In this regard, the average flow stress from a strain of $4.5-8 \%$ is plotted against $\lambda / d$ in Fig. 2(c). It can be seen that the average flow stresses of both the laminated structure and the equiaxed grain structure first increase with decreasing $\lambda / d$, reaching a maximum at a critical $\lambda / d$, and then decrease when $\lambda / d$ becomes even smaller. As indicated from previous research $[9,12]$, this behavior is due to a transition of dominant deformation mechanisms from dislocationmediated plasticity to GB-associated plasticity. It is also observed that the critical size $\lambda_{\text {crit }}(12.5 \mathrm{~nm})$ in the laminated structure is much smaller than the critical size $d_{\text {crit }}(20 \mathrm{~nm})$ in the equiaxed 

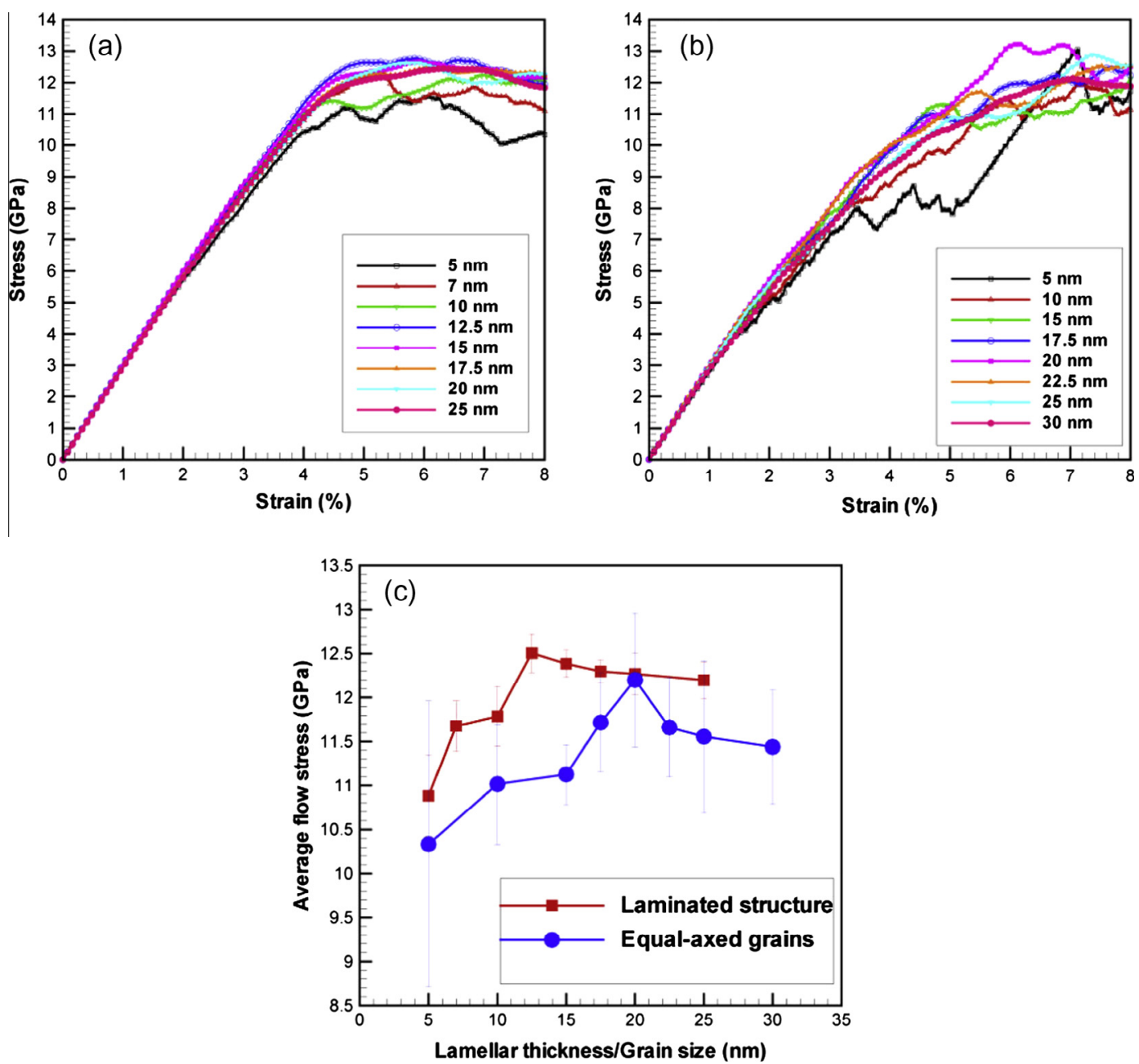

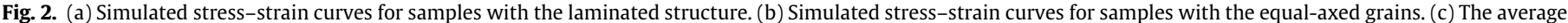
flow stress vs. the lamellar thickness/grain size.

grain structure. Moreover, the strength of the laminated structure was found to be higher than that of the equiaxed grain structure at the same length scale.

The observed flow stress behaviors and the mechanisms behind the plastic deformation can be disclosed through an atomistic analysis of the deformed configurations. First, the rotations of grains at a triple junction area were checked and compared at the same length scale for both the laminated structure and the equiaxed grained structure. Figs. 3 and 4 show these comparisons for $\lambda / d=5 \mathrm{~nm}$ and $25 \mathrm{~nm}$, respectively. As indicated, the misorientation angle between grains 1 and 4 is $10^{\circ}$, while the misorientation angle between grains 2 and 4 is $15^{\circ}$ before deformation for both structures. After $8 \%$ tensile deformation, the corresponding misorientation angles change to $6^{0}$ and $10^{\circ}$ for the laminated structure with $\lambda=5 \mathrm{~nm}$, and reduce to $0^{0}$ and $3^{0}$ for the equiaxed grain structure with $d=5 \mathrm{~nm}$. The grain growth and the grain coalescence induced by grain rotations are observed for equiaxed grain structure with $d=5 \mathrm{~nm}$, where the initial four grains are nearly growing into one big grain. However, these misorientation angles do not change during the tensile deformation for the laminated structure with $\lambda=25 \mathrm{~nm}$, and reduce to $8^{\circ}$ and $10^{\circ}$ for the equal-axed grained structure with $d=25 \mathrm{~nm}$.

For the same structure, the smaller the $\lambda / d$, the larger the grain rotation, which is due to the increased GB activities associated with smaller $\lambda / d$ and a change in the dominant deformation mechanism as suggested in previous research [9,12], e.g., from dislocation-mediated dominating plasticity to GB-associated dominating plasticity. Of course, this is not a sharp transition, and even in the case of $\lambda / d=5 \mathrm{~nm}$, a few dislocation activities are still seen to occur and the simultaneous operations of two slip systems in each grain are usually required due to the deformation compatibility at GBs. More interestingly, much less grain rotations are observed for the laminated structure when compared to the equiaxed grain structure at the same length scale. This indicates that GB activities can be inhibited by the laminated structure at the samle length scale, which results in enhanced strength and smaller critial size, as shown in Fig. 2(c).

In order to obtain the detailed strain distribution and the strain partitioning between GB atoms and non-GB atoms, a local equivalent strain was calculated using the atomic local strain tensor: $\varepsilon_{e V}=\sqrt{3 J_{2}(\varepsilon)}$, where $J_{2}(\varepsilon)$ is the second deviatoric strain invariant. The local strain tensor is not too difficult to define when the reference state at $0 \%$ strain is known, which can be calculated as a best fit of the local affine transformation using the all local neighbor atom's information, through running a least-square fitting procedure $[36,38]$. This equivalent strain contour and mapping are pretty useful in MD simulations, allowing us to directly not only obtain the strain partitioning but also visualize microstructure with color-encoding strain fields, such as dislocations, GBs, stacking faults (SFs), voids, and cracks [36,38].

Fig. 5(a) and (d) shows the local equivalent strain contours after $8 \%$ tensile loading for the samples at the same length scale $(\lambda / d=25 \mathrm{~nm})$ with the laminated structure and the equiaxed grain 
(a)

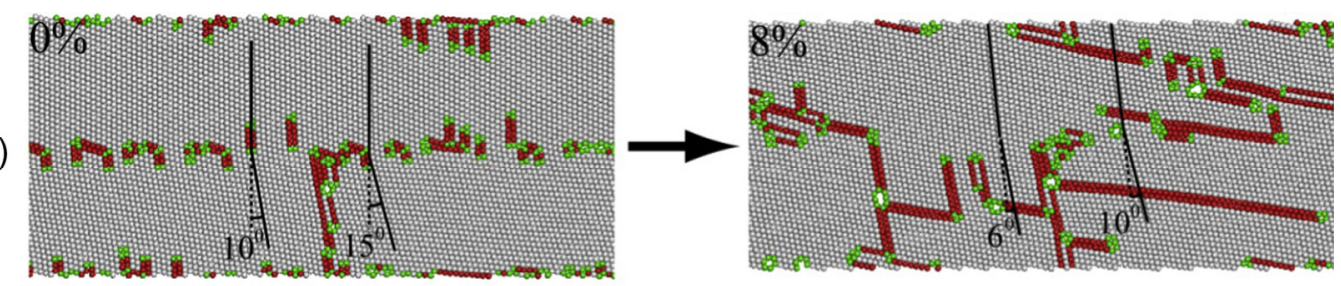

(b)
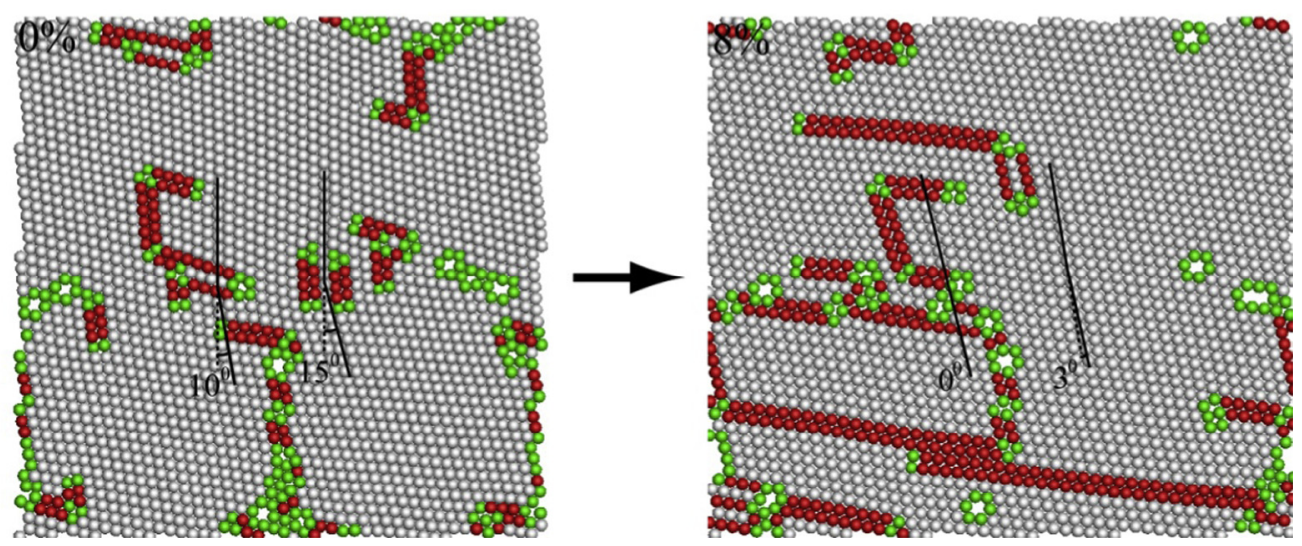

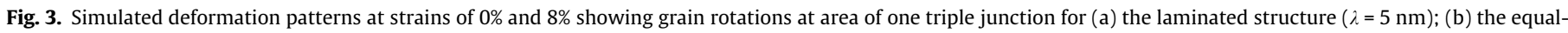
axed grain structure $(d=5 \mathrm{~nm})$.

(a)

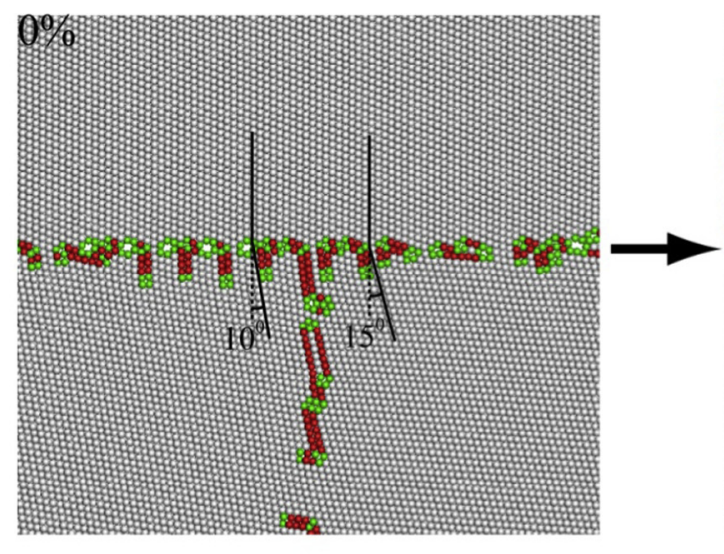

(b)

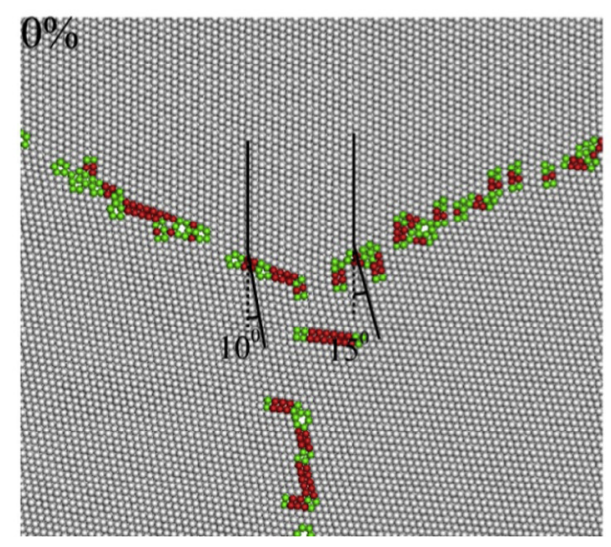

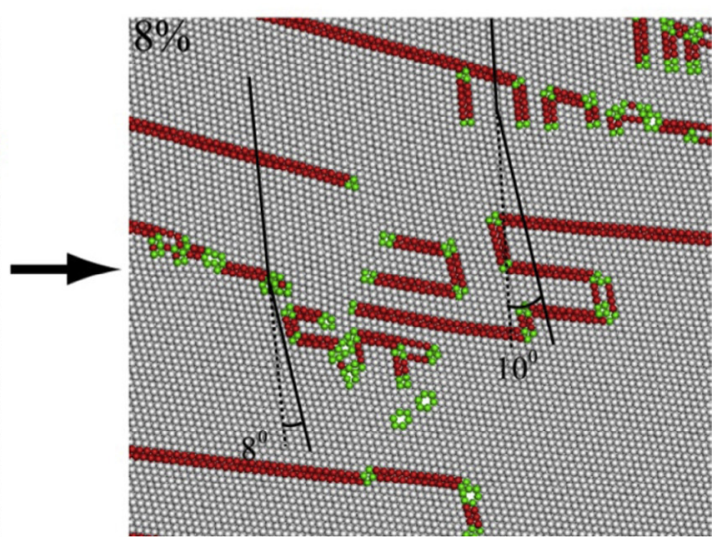

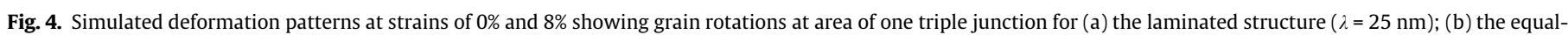
axed grain structure $(d=25 \mathrm{~nm})$.

structure, respectively. Fig. 5(b) and (c) are the corresponding amplified configurations from Fig. 5(a). The initial GB atoms at $0 \%$ strain are also shown separately by black color. The detailed observations are as follows: (i) two of slip planes are activated, which allows the emission and propagation of partial dislocations from one side of GBs to the opposite side, leaving SFs behind; (ii) large local strains are observed and accumulated at GB and SF areas; (iii) in the laminated structure, most of partial dislocations are absorbed and blocked by the horizontal lamellar boundaries, indicating the lamellar thickness to be the controlling length scale 
(a)

(b)

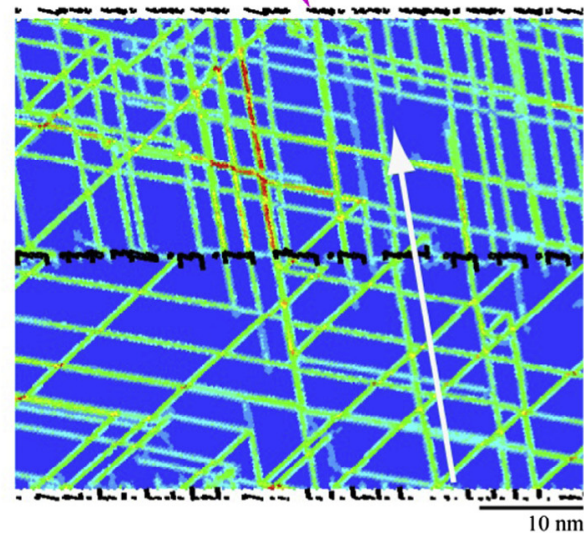

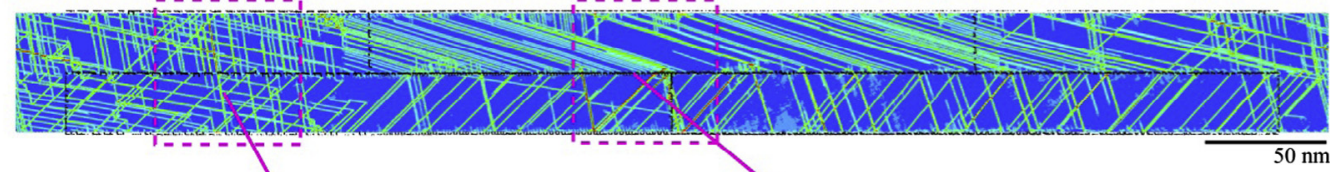

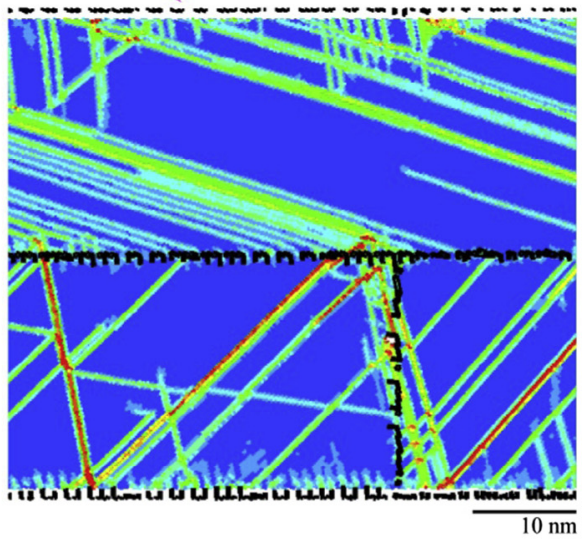

(d)

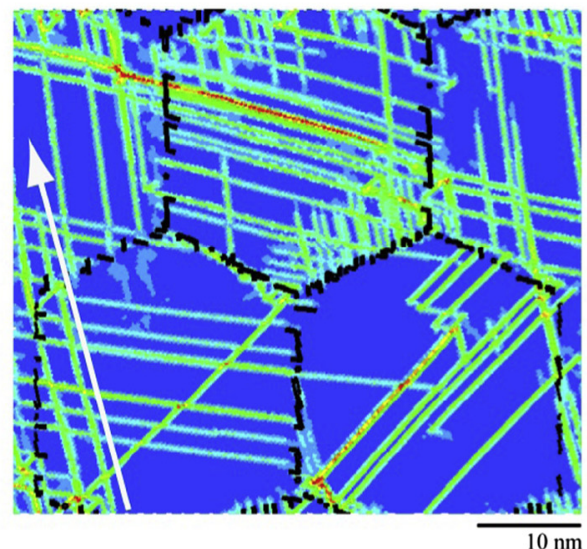

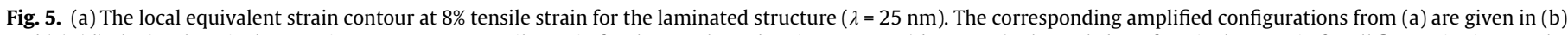

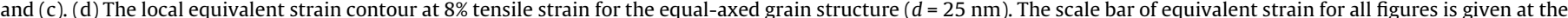
right side of (d). White arrows are also given in (b) and (d) indicating typical dislocation transmission.

for strengthening; (iv) a small amount of dislocation transmissions across LAGBs are also observed in both the laminated structure and the equiaxed grain structure due to small misorientation angles. As suggested in previous research [39], transmission of dislocations across the GB is a complicated process, including the incoming dislocations merging into the GB, dissociating into other dislocations in the adjacent grains, and leaving a GB dislocation and creating a step at the GB. Both experimental and atomistic simulation efforts have been reviewed recently to illustrate the details of slip transmission at GBs [40], and three criteria have been proposed to predict the favorable slip system for slip transmission: (i) the slip system geometry approach (the angle between the incoming and outgoing slip planes in the adjacent grains across the GB should be as small as possible); (ii) the stressbased approach; (iii) and the residual GB dislocation-based approach $[40,41]$. Based on the first criterion, LAGBs with small misorientation angles are more prone to dislocation transmission, which is consistent with the current results.

In order to obtain the plastic deformation, we relaxed the samples to zero stress after $8 \%$ tension, and then the average local equivalent plastic strains were obtained based on the relaxed configuration. Fig. 6 shows the average local equivalent plastic strains for all atoms and GB atoms in both the laminated structure and the

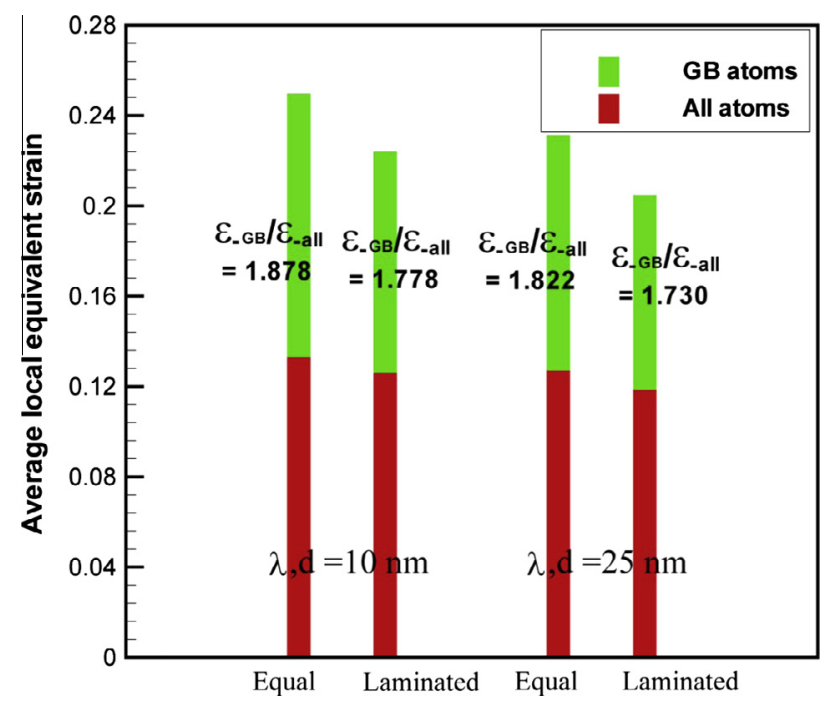

Fig. 6. Simulated average local equivalent plastic strains of both the laminated structure and the equal-axed grain structure for two different $\lambda / d(10$ and $25 \mathrm{~nm}$ ) values. 
equiaxed grain structure with the same length scale $(\lambda / d=10$ and $25 \mathrm{~nm}$ ). It is shown that the average local equivalent plastic strains for both all atoms and GB atoms in the laminated structure are smaller than those in the equiaxed grain structure for both values of $\lambda / d$ (10 and $25 \mathrm{~nm}$ ), indicating that the laminated structure is more effective in resisting plastic deformation. More importantly, the normalized average local equivalent plastic strain for GB atoms
$\left(\varepsilon_{G B} / \varepsilon_{\text {all }}\right)$ in the laminated structure is also smaller than that in the equiaxed grain structure. $\varepsilon_{G B} / \varepsilon_{\text {all }}$ is actually an indication of strain partitioning between GB atoms and non-GB atoms, and is a comparison between GB activities (GB sliding, grain rotation and atomic shuffling at GBs [42]) and dislocation activities. So, Fig. 6 also indicates that GB activities can be inhibited by the laminated structure at the same length scale.

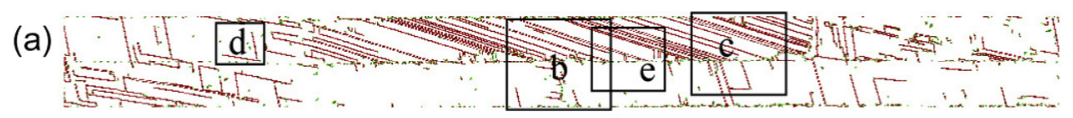

(b)

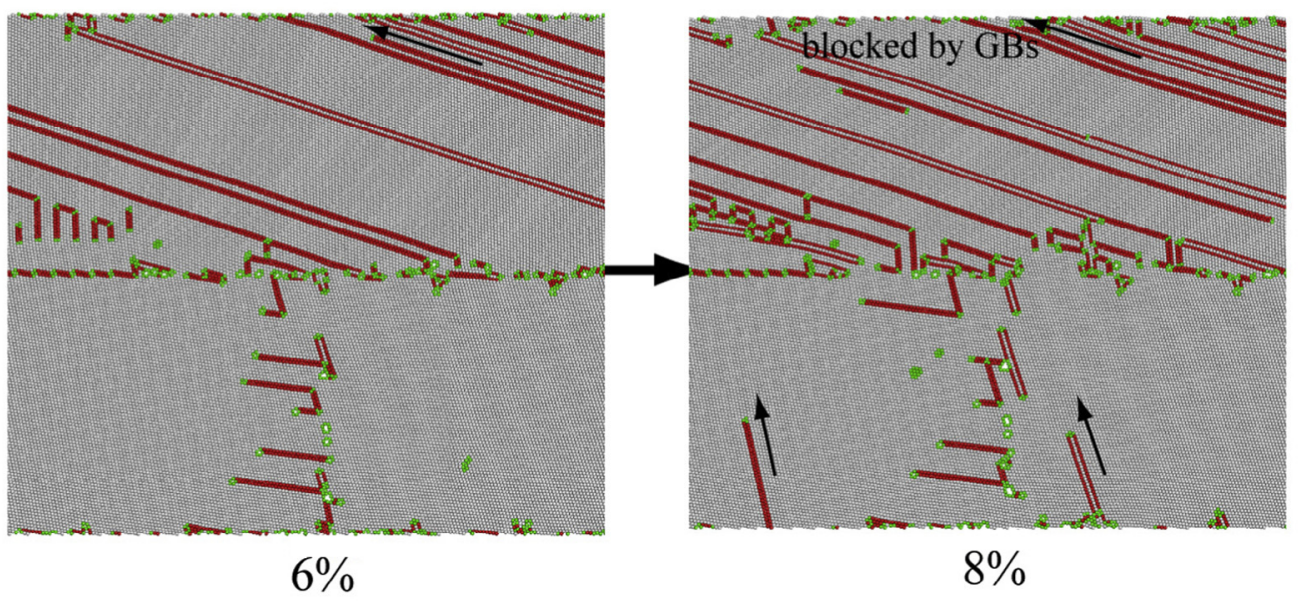

(c)

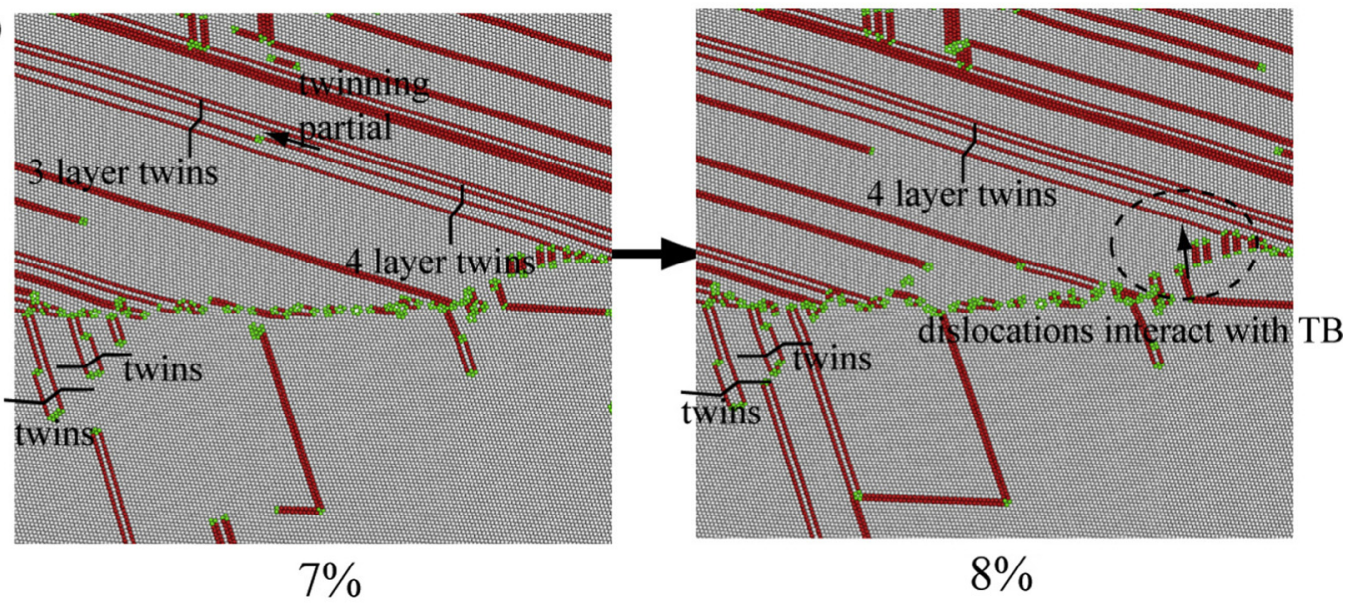

(d)

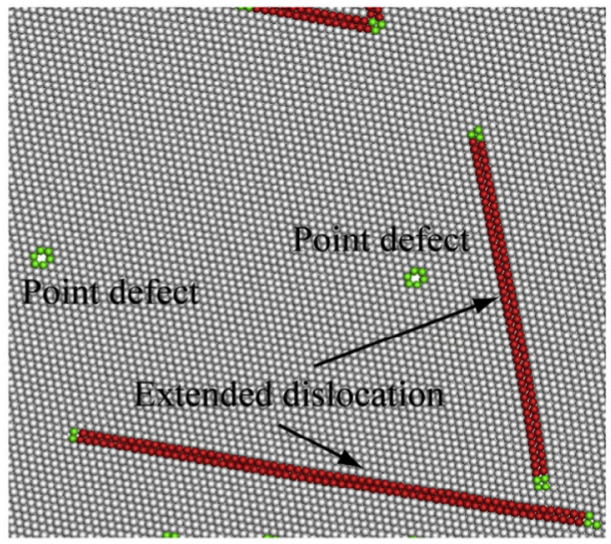

$8 \%$ (e)

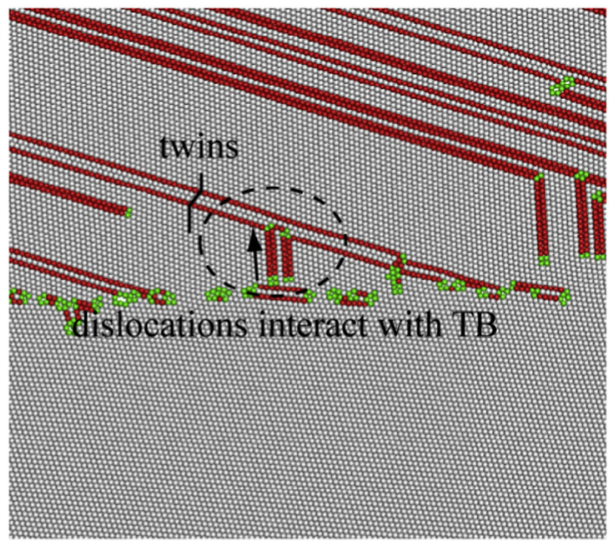

$8 \%$

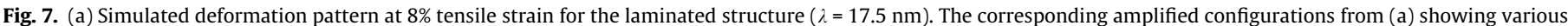

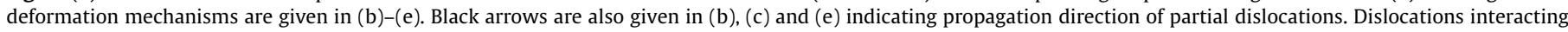
with TBs are marked by dashed ellipse. 
Apparently, GB activities can be inhibited by the laminated structure at the samle length scale compared to the equiaxed grain structure, which is mainly due to the different grain structures and the defect structures at the boundaries. Moreover, according to previous research $[22,43,44]$, dislocations can not easily move from one layer to another layer in bi-metal multilayered system in spite of continuity of slip mostly due to the in-plane internal strains and stresses. These internal strains and stresses are induced by matching of the two adjacent lattices with misfit dislocations at the interfaces, and the maximum strength for interface crossing mechanism at the critical layer thickness is highly related to the internal stresses. In our case, LAGBs are actually dislocation walls with numerous misfit dislocations due to the small misorientation angles. These misfit dislocations at the interfaces of the laminated structure should create internal stresses at the long interfaces similar to the bi-metal multilayered system and the dependence of internal stresses on the lamellar thickness for the laminated structure should be more sensitive than the polycrystal model with randomly oriented equiaxed grains. As indicated [22,43,44], one of important effects on strengthening derives from the internal strains and stresses, which gives another reason why the laminated structures possess enhanced strength compared to the equiaxed grain structure.

Besides GB activities, other atomistic deformation mechanisms are also shown in Fig. 7 for a typical laminated structure $(\lambda=17.5 \mathrm{~nm})$. The overall simulated deformation pattern at the overall tensile strain of $8 \%$ is shown in Fig. 7(a). Fig. 7(b)-(e) displays the corresponding amplified configurations from Fig. 7(a) showing the various deformation mechanisms other than GB activities. Two snapshots at different strains were given to show the dynamic process for some of deformation mechanisms. It can be summarized as follows: (i) partial dislocations nucleated from GBs are absorbed and blocked by the lamellar boundaries or interact with SFs from other slip planes (the dominating deformation mechanisms, Fig. 7(b)); (ii) formation of deformation twins and partial dislocations from other slip systems interact with the formed TBs (Fig. 7(c) and (e)); (iii) nucleation of extended dislocations and point defects inside grain interior (Fig. 7(d)); (iv) partial dislocation transmission through LAGBs as observed from the local equivalent strain contours (Fig. 5(b) and (d)). According to previous research [20,45], the SFs as well as dislocation locks associated with dislocation/SF reactions could be generated by the first deformation mechanism (the dominant one), those locks are immobile and cause the work hardening. The second deformation mechanism also generates SFs and dislocation locks, causing the work hardening, except these locks are associated with dislocation/TB interactions instead of dislocation/SF reactions [20,45].

Of course, some deformation behaviors (such as diffusion effects) might be limited due to the short time scale in MD simulations. Thus, one can not directly compare the stress-strain response from MD simulations with the experimental curves due to the strain rate effects. However, the strengths for different grain sizes/TBS based on MD simulations only can be compared, and the size effects on the strength of nanostructured metals [9,17] could be investigated by MD simulations when the simulation cells are carefully designed and the same crystallographic orientations of all grains are retained. Thus, the MD simulations might provide insights for experiments. As indicated in previous research $[40,41]$, LAGBs are less effective in resisting plastic deformation than high-angle grain boundaries (HAGBs) due to the higher possibility for dislocation transmission. However, from previous experimental results [29], the hardness of the nano-laminated $\mathrm{Ni}$ was found to lie on the extrapolated Hall-Patch line based on other experimental data by taking the lamellar thickness as the equivalent grain size, indicating that the nano-laminated boundaries with LAGBs are as effective in strengthening as the conventional HAGBs in equiaxed nano-grains. Combining these two observations together, one conclusion can be easily drawn that the laminated structure should have enhanced strength compared to the equiaxed grain structure when both structures have LAGBs and the same length scale, which is consistent with our results and validates our simulation results.

\section{Concluding remarks}

In the present study, a series of large-scale MD simulations have been conducted to find out the critical length scale and the underlying atomistic deformation mechanisms for both the laminated structure and the equiaxed grain structure. The main findings are summarized as follows:

(1) The laminated structure was found to be more effective in resisting plastic deformation. Smaller critical size and enhanced strength were observed in the laminated structure when compared to the equiaxed grain structure.

(2) GB activities were found to be inhibited by the laminated structure at the same length scale by checking the rotations of grains and the equivalent strain partitioning in GB atoms. The laminated structure is also found to be more mechanical stable.

(3) Besides GB activities, formation of extended dislocations, formation of deformation twins, partial dislocations interacting with formed TBs, partial dislocation blocking by and even transmission across LAGBs were also found to play important roles in plastic deformation of the nanolaminated structure.

The results in the present paper should provide insights to design the microstructures for reinforcing the mechanical properties in nanostructured metals.

\section{Acknowledgments}

This work was supported by the NSFC under Grants No. 11222224, No. 11472286, and No. 11021262; and the National Key Basic Research Program of China under Grants No. 2012СB932203 and No. 2012СB937500. The simulations reported in the present study were performed at Supercomputing Center of Chinese Academy of Sciences.

\section{References}

[1] M. Dao, L. Lu, R.J. Asaro, J.T.M. De Hosson, E. Ma, Acta Mater. 55 (2007) 40414065.

[2] M.A. Meyers, A. Mishra, D.J. Benson, Prog. Mater. Sci. 51 (2006) 427-556.

[3] N. Tsuji, Y. Ito, Y. Saito, Y. Minamino, Scripta Mater. 47 (2002) 893-899.

[4] R.Z. Valiev, T.G. Langdon, Prog. Mater. Sci. 51 (2006) 881-981.

[5] Y.S. Li, N.R. Tao, K. Lu, Acta Mater. 56 (2008) 230-241.

[6] A.P. Zhilyaev, T.G. Langdon, Prog. Mater. Sci. 53 (2008) 893-979.

[7] X.L. Wu, P. Jiang, L. Chen, F.P. Yuan, Y.T. Zhu, Proc. Nat. Acad. Sci. USA 111 (2014) 7197-7201.

[8] T.H. Fang, W.L. Li, N.R. Tao, K. Lu, Science 331 (2011) 1587-1590.

[9] J. Schiotz, K.W. Jacobsen, Science 301 (2003) 1357-1359.

[10] K. Kadau, T.C. Germann, P.S. Lomdahl, B.L. Holian, D. Kadau, P. Entel, M. Kreth, F. Westerhoff, D.E. Wolf, Science 35A (2004) 2719-2723.

[11] T.G. Nieh, J.G. Wang, Intermetallics 13 (2005) 377-385.

[12] J.B. Jeon, B.J. Lee, Y.W. Chang, Scripta Mater. 64 (2011) 494-497.

[13] C.C. Koch, J. Narayan, MRS Proc., vol. 634, Cambridge University Press, London, England, 2000.

[14] N.Q. Vo, R.S. Averback, P. Bellon, A. Caro, Scripta Mater. 61 (2009) 76-79.

[15] Z. Qi, Sun, F. Zhu, Z. Wang, D. Peng, C. Wu, Surf. Coat. Technol. 205 (2011) 3692-3697.

[16] L. Lu, X. Chen, X. Huang, K. Lu, Science 323 (2009) 607-610.

[17] X.Y. Li, Y.J. Wei, L. Lu, K. Lu, H.J. Gao, Nature 464 (2010) 877-880.

[18] F.P. Yuan, X.L. Wu, Phys. Rev. B 86 (2012) 134108.

[19] F.P. Yuan, X.L. Wu, J. Appl. Phys. 113 (2013) 203516.

[20] Y.T. Zhu, X.Z. Liao, X.L. Wu, Prog. Mater. Sci. 57 (2012) 1-62.

[21] A. Misra, J.P. Hirth, R.G. Hoaland, Acta Mater. 53 (2005) 4817-4824. 
[22] R.G. Hoaland, R.J. Kurtz, C.H. Henager, Scripta Mater. 50 (2004) 775-779.

[23] J. Wang, R.G. Hoagland, J.P. Hirth, A. Misra, Acta Mater. 56 (2008) 5685-5693.

[24] J. Wang, R.G. Hoagland, A. Misra, Scripta Mater. 60 (2009) 1067-1072.

[25] Y.M. Wang, J. Li, A.V. Hamza, T.W. Barbee Jr., Proc. Nat. Acad. Sci. USA 104 2007) 11155-11160.

[26] Y.P. Li, G.P. Zhang, W. Wang, J. Tan, S.J. Zhu, Scripta Mater. 57 (2007) 117-120.

[27] J.Y. Zhang, X. Zhang, G. Liu, G.J. Zhang, J. Sun, Mater. Sci. Eng. A-Struct. Mater. Prop. Microstruct. Process. 528 (2011) 2982-2987.

[28] H.M. Wen, Y.H. Zhao, T.D. Topping, D. Ashford, R.B. Figueiredo, C. Xu, T.G. Langdon, E.J. Lavernia, Adv. Eng. Mater. 14 (2012) 185-194.

[29] X.C. Liu, H.W. Zhang, K. Lu, Science 342 (2013) 337-340.

[30] V. Yamakov, D. Wolf, S.R. Phillpot, A.K. Mukherjee, H. Gleiter, Nat. Mater. 1 (2002) 45-48.

[31] H. Van Swygenhoven, P.M. Derlet, A.G. Froseth, Nat. Mater. 3 (2004) 399-403.

[32] Y.N. Osetsky, D. Rodney, D.J. Bacon, Phil. Mag. 86 (2006) 2295-2313.

[33] F.P. Yuan, X.L. Wu, Comput. Mater. Sci. 85 (2014) 8-15.
34] D. Farkas, L. Patrick, Phil. Mag. 89 (2009) 3435-3450.

[35] W.Z. Han, Q. An, S.N. Luo, T.C. Germann, D.L. Tonks, W.A. Goddard III, Phys. Rev. B 85 (2012) 024107.

36] X.M. Liu, F.P. Yuan, Y.G. Wei, Appl. Surf. Sci. 279 (2013) 159-166.

37] Y. Mishin, D. Farkas, M.J. Mehl, D.A. Papaconstantopoulos, Phys. Rev. B 59 (1999) 3393-3407.

[38] F. Shimizu, S. Ogata, J. Li, Mater. Trans. 48 (2007) 2923-2927.

39] H. Jang, D. Farkas, Mater. Lett. 61 (2007) 868-871.

[40] D.E. Spearot, M.D. Sangid, Curr. Opin. Solid State Mater. Sci. 18 (2014) 188195.

41] T.C. Lee, I.M. Robertson, H.K. Birnbaum, Philos. Mag. A-Phys. Condens. Mat. Struct. Def. Mech. Prop. 62 (1990) 131-153.

[42] H. Van Swygenhoven, P.M. Derlet, A. Hasnaoui, Phys. Rev. B 66 (2002) 024101.

[43] B. Shoykhet, M.A. Grinfeld, P.M. Hazzledine, Acta Mater. 46 (1998) 3761-3766

[44] H.M. Zbib, C.T. Overman, F. Akasheh, D. Bahr, Int. J. Plast. 27 (2011) 1618-1639.

[45] L. Lu, Z.S. You, K. Lu, Scripta Mater. 66 (2012) 837-842. 